To be Submitted to ApJ

Preprint typeset using $\mathrm{LAT}_{\mathrm{E}} \mathrm{X}$ style emulateapj v. 08/22/09

\title{
GENERAL RELATIVISTIC MAGNETOHYDRODYNAMIC SIMULATIONS OF THE HARD STATE AS A MAGNETICALLY-DOMINATED ACCRETION FLOW
}

\author{
P. Chris Fragile \\ Department of Physics \& Astronomy, College of Charleston, Charleston, SC 29424; fragilep@cofc.edu \\ AND \\ DAVID L. MEIER \\ Jet Propulsion Laboratory, California Institute of Technology, Pasadena, CA 91109; David.L.Meier@jpl.nasa.gov \\ (Dated: IATEX-ed July 26, 2021) \\ To be Submitted to ApJ
}

\section{ABSTRACT}

We present one of the first physically-motivated two-dimensional general relativistic magnetohydrodynamic (GRMHD) numerical simulations of a radiatively-cooled black-hole accretion disk. The fiducial simulation combines a total-energy-conserving formulation with a radiative cooling function, which includes bremsstrahlung, synchrotron, and Compton effects. By comparison with other simulations we show that in optically thin advection-dominated accretion flows, radiative cooling can significantly affect the structure, without necessarily leading to an optically thick, geometrically thin accretion disk. We further compare the results of our radiatively-cooled simulation to the predictions of a previously developed analytic model for such flows. For the very low stress parameter and accretion rate found in our simulated disk $\left(\alpha \approx 0.003, \dot{M} / \dot{M}_{E d d} \approx 5 \times 10^{-6}\right)$, we closely match a state called the "transition" solution between an outer advection-dominated accretion flow and what would be a magnetically-dominated accretion flow (MDAF) in the interior. The qualitative and quantitative agreement between the numerical and analytic models is quite good, with only a few well-understood exceptions. According to the analytic model then, at significantly higher $\alpha$ or $\dot{M}$, we would expect a full MDAF to form.

The collection of simulations in this work also provide important data for interpreting other numerical results in the literature, as they span the most common treatments of thermodynamics, including simulations evolving: 1) the internal energy only; 2) the internal energy plus an explicit cooling function; 3) the total energy without cooling; and 4) total energy including cooling. We find that the total energy formulation is a necessary prerequisite for proper treatment of radiative cooling in MRI accretion flows, as the internal energy formulation produces a large unphysical numerical cooling of its own. We also find that the relativistic cooling functions must be handled carefully numerically in order to avoid equally unphysical heating or cooling runaways.

Subject headings: accretion, accretion disks — black hole physics — galaxies: active — MHD relativity - X-rays: stars

\section{INTRODUCTION}

The process by which turbulent accretion flows around black holes develop large scale magnetic fields that can drive collimated jet outflows is still poorly understood. Much of what we know comes from observations of Xray binaries (XRBs) (summarized by Fender et al. 2004), largely because the rapid variability in these stellarmass systems allow for observations of state changes on timescales of days to at most a few years, whereas state changes are rarely observed for AGN. In XRBs, jets are associated with both the Hard and Soft accretion states (McClintock \& Remillard 2006), although their characteristics in the two states are quite different. Hard state jets are very steady, potentially lasting for weeks, whereas jets produced in the transition to the high accretion-rate Soft state can be explosive and short-lived. One possible interpretation of this phenomenology (Fender et al. 2004) is that accreting black holes produce jets most of the time, with the jet speed being a function of the accretion rate. Objects in the Hard state produce slow $(v \sim 0.3 c)$ jets, while Soft-state objects produce fast jets with flat-space Lorentz factors
$W \equiv\left(1-v^{2} / c^{2}\right)^{-1 / 2} \sim 10$ that are comparable with jets in higher luminosity AGN (e.g., FR II radio sources). The explosive jet, in this picture, is simply the bow shock of a new fast jet interacting with a previously-existing slow jet as the source's accretion rate temporarily, and rapidly, increases toward the Eddington limit.

Recent numerical simulations of non-radiative magnetohydrodynamic (MHD) flows that are unstable to the magneto-rotational instability (MRI) have been shown to produce jetted outflows (Hirose et al. 2004; McKinney 2006). In those simulations the mechanism involves the development of a magnetically-dominated region close to the black hole and rotation axis, where the magnetic field orders itself into a helical, rotating structure that drives the jet. At the present time, however, it is not clear if these results fit the phenomenology described above. The problem is that non-radiative MRI simulations should be the proper theoretical counterpart to the Hard state, but the simulations produce relativistic jets instead of the slow type jets associated with the Hard state.

One of the present authors has suggested (Meier 2005. 2008, 2009) that, at moderately low accretion rates 
(where turbulent, advection-dominated accretion flows or ADAFs should occur), the inflow inside a radius $R_{1} \sim 100 r_{G}=100 \mathrm{GM} / \mathrm{c}^{2}$ should develop a black hole magnetosphere structure similar to the force-free ones studied recently by Tomimatsu \& Takahashi (2001); Uzdensky (2004, 2005). In this picture, closed field lines connecting the disk at $R_{1}$ with the event horizon could funnel ionized plasma toward the black hole, creating a magnetically-dominated accretion flow or MDAF. Open field lines anchored near $R_{1}$, on the other hand, could drive a jet outflow with a speed set by the dynamical timescale near $R_{1}\left[\sim\left(G M / R_{1}\right)^{1 / 2} \sim 0.1 c\right]$. A further strength of this model is that a relatively large magnetosphere might help explain why quasi-periodic oscillations in the Hard state are observed in the Hertz range, rather than $\mathrm{kHz}$.

Our suggested mechanism for MDAF/magnetosphere formation is radiative cooling in the previously-supposed, radiatively-inefficient ADAF. Cooling would lower the plasma pressure and decrease the disk vertical scale height, both of which lead to a dramatic increase in the dominance of magnetic stresses (ratio of magnetic to gas pressure greater than unity). Strongly-magnetized plasmas are much more stable to the MRI, leading to a decrease in turbulence and a more ordered magnetic field. This is precisely the same process that occurred in the Hirose et al. (2004) and McKinney (2006) simulations, but now at a radius of $\sim 100 r_{G}$ instead of a few.

A critical assumption in this picture, though, is that the entire plasma (electrons and ions) cools. This is in contrast to current accretion theory, which asserts that whenever the flow enters a hot, Hard X-ray state, the transfer of thermal energy between ions and electrons is inefficient, leading to a two-temperature, optically thin ADAF with cool electrons and hot ions. However, some theoretical and numerical work has suggested this may not be the case (e.g. Begelman \& Chiueh 1988; Sharma et al. 2007). There is also some observational evidence that efficient energy transfer from ions to electrons must occur even when a black hole is in a Hard X-ray state. Monitoring of many black-hole candidate sources shows that they can be found in the Soft state at bolometric luminosities lower than in the maximum Hard state. This appears to be especially true in Hard states where a strong, steady jet is produced (e.g., the plateau state). Sources at the top right of Fig. 7 of Fender et al. (2004) (the "FBG diagram") are quite hard and yet quite luminous. As a concrete example, Cygnus X-1 produces $90 \%$ or more as much bolometric luminosity in the Hard state as in its Soft state (McConnell et al. 2002). This is hardly "radiatively inefficient" accretion by any standard. The only truly inefficient state might be the Quiescent state, of which the black-hole candidate A0620-00 and the Galactic center black hole Sgr A* may be examples.

Our goal in this paper is not to resolve the controversy of whether or not Hard state objects radiate efficiently, but rather to investigate how they will behave if they do. We proceed by performing general relativistic MHD simulations of MRI-unstable black hole accretion flows, with two key differences from previous investigations: we include in the energy equation a plausible high-temperature cooling function that is relevant for such flows, and we assume that electrons and ions are sufficiently thermally coupled that cooling of the former also cools the latter, keeping $T_{i} \approx T_{e}$. For completeness, we actually compare four different classes of numerical models: one that evolves internal energy without including cooling, one that evolves internal energy and includes cooling, one that conserves total energy but does not include cooling, and one that conserves total energy and includes cooling. These four classes of models span most of the simulations that have been carried out to date by other authors, and expands significantly on what has been done thus far with simulations involving physical cooling mechanisms. Our paper is unique in that it gives the first direct comparison of all four using a single numerical code and which, we believe, is the first to investigate numerically the triggering of state transitions in cooled black hole accretion flows.

\section{NUMERICAL METHODS}

This work is carried out using the Cosmos ++ astrophysical MHD code (Anninos et al. 2005). Cosmos ++ includes several schemes for solving the MHD equations, including a traditional artificial viscosity (AV) scheme and a new extended artificial viscosity (eAV) method. The AV scheme is based on an internal-energy-evolving (entropy-conserving) scheme, whereas the eAV scheme is a hybrid dual energy scheme that solves both the internal and total energy equations. The eAV scheme has the obvious advantage that it conserves total energy; it is also potentially more accurate than other fully conservative schemes in tracking the internal energy of the gas because of the dual treatment. Artificial viscosity based schemes, which both of these are, further have the advantage that they are simpler to deal with when it comes to including extra physics such as the radiative cooling being added in this work. Furthermore, the combination of AV and eAV methods allows us to directly compare, within a single numerical code, the effects of including realistic heating and cooling processes in the evolution of MRI turbulent accretion disks.

Cosmos ++ has options to solve the MHD equations in either a Newtonian or general relativistic framework. Here the general relativistic form is used. In writing our equations we use the standard notation in which fourand three-dimensional tensor quantities are represented by Greek and Latin indices, respectively, and repeated indices imply summation. The equations of mass conservation, momentum conservation, and magnetic induction, common to both numerical methods used in this work, have the form

$$
\begin{aligned}
\partial_{t} D+\partial_{i}\left(D V^{i}\right)= & 0, \\
\partial_{t} S_{j}+\partial_{i}\left(S_{j} V^{i}\right)= & \frac{1}{4 \pi} \partial_{t}\left(\sqrt{-g} B_{j} B^{0}\right)+\frac{1}{4 \pi} \partial_{i}\left(\sqrt{-g} B_{j} \mathcal{B}^{i} 2\right) \\
& +\left(\frac{S^{\mu} S^{\nu}}{2 S^{0}}-\frac{\sqrt{-g}}{8 \pi} B^{\mu} B^{\nu}\right) \partial_{j} g_{\mu \nu} \\
& -\sqrt{-g} \partial_{j}\left(P+P_{B}+Q\right) \\
& +\Gamma W u_{j} \Lambda, \\
\partial_{t} \mathcal{B}^{j}+\partial_{i}\left(\mathcal{B}^{j} V^{i}\right)= & \mathcal{B}^{i} \partial_{i} V^{j}+g^{i j} \partial_{i} \psi \\
\partial_{t} \psi+c_{h}^{2} \partial_{i} \mathcal{B}^{i}= & -\frac{c_{h}^{2}}{c_{p}^{2}} \psi,
\end{aligned}
$$


where $g_{\mu \nu}$ is the 4-metric, $g$ is the 4-metric determinant, $W=\sqrt{-g} u^{0}$ is the relativistic boost factor, $D=W \rho$ is the generalized fluid density, $V^{i}=u^{i} / u^{0}$ is the transport velocity, $u^{\mu}=g^{\mu \nu} u_{\nu}$ is the fluid 4-velocity, $S_{\mu}=$ $W\left(\rho h+2 P_{B}\right) u_{\mu}$ is the covariant momentum density, $P$ is the fluid pressure, $Q$ is the artificial viscosity used for shock capturing, $\Gamma$ (without subscripts or superscripts) is the adiabatic index, $c_{h}$ and $c_{p}$ are coefficients to determine the strength of the hyperbolic and parabolic pieces of the divergence cleanser, and $\Lambda(\rho, T, H, B)$ is the cooling function of a gas with density $\rho$, temperature $T$, temperature scale height $H$, and magnetic field strength $B$, as described in detail in the next section. (With indices, $\Gamma$ indicates the geometric connection coefficients of the metric.) There are two representations of the magnetic field in our equations: $B^{\mu}$ is the 4-vector of the magnetic field, which can be defined in terms of the dual of the Faraday tensor $\left(B^{\mu} \equiv u_{\nu}{ }^{*} F^{\mu \nu}\right)$, and $\mathcal{B}^{i}=W\left(B^{i}-B^{0} V^{i}\right)$ is the boosted magnetic field 3 -vector, where $B^{0}$ is recovered from the orthogonality condition $B^{\mu} u_{\mu}=0$,

$$
B^{0}=-\frac{W}{g}\left(g_{0 i} \mathcal{B}^{i}+g_{i j} \mathcal{B}^{j} V^{i}\right) .
$$

The magnetic pressure is $P_{B}=\|B\|^{2} / 8 \pi=$ $g_{\mu \nu} B^{\mu} B^{\nu} / 8 \pi$. We have assumed an equation of state of the form $P=(\Gamma-1) \rho \epsilon$ with $\epsilon$ being the internal energy. We use the scalar $Q$ from Anninos et al. (2005) with $k_{q}=2.0$ and $k_{l}=0.3$. We fix the divergence cleanser coefficients to be $c_{h}=c_{\mathrm{cfl}} \Delta x_{\mathrm{min}} / \Delta t$ and $c_{p}^{2}=c_{h}$, where $c_{\mathrm{cfl}}=0.5$ is the Courant coefficient, $\Delta x_{\min }$ is the minimum covariant zone length, and $\Delta t$ is the evolution timestep.

Both computational schemes also solve the internal energy equation in the form

$$
\begin{aligned}
\partial_{t} E+\partial_{i}\left(E V^{i}\right)= & -P \partial_{t} W-(P+Q) \partial_{i}\left(W V^{i}\right) \\
& +W \Lambda(\rho, T, H, B)
\end{aligned}
$$

where $E=W e=W \rho \epsilon$ is the generalized internal energy density. The temperature $T$ in the cooling function is recovered from the internal energy of the gas using the ideal gas law

$$
T=(\Gamma-1) e /(k n)
$$

where $n=\rho /\left(\mu m_{H}\right)$ is the number density of the gas and we use $\mu=1.69$.

Additionally the hybrid dual energy scheme solves the following total energy equation

$$
\begin{aligned}
\partial_{t} \mathcal{E} & +\partial_{i}\left(\mathcal{E} V^{i}\right)=\Sigma^{0}-\partial_{i}\left(F^{i}\right) \\
& +\left(\frac{W^{2} \Gamma}{\sqrt{-g}}+\sqrt{-g} g^{00}(\Gamma-1)\right) \Lambda(\rho, T, H, B),
\end{aligned}
$$

where the total energy $\mathcal{E}$ is defined as

$$
\begin{aligned}
\mathcal{E}=\sqrt{-g} T^{00}= & \frac{W^{2}}{\sqrt{-g}}\left(\rho h+2 P_{B}\right)+\sqrt{-g} g^{00}\left(P+P_{B}(9)\right. \\
& -\frac{1}{4 \pi} \sqrt{-g} B^{0} B^{0},
\end{aligned}
$$

the curvature source term is

$$
\Sigma^{0}=-\sqrt{-g} T^{\alpha \beta} \Gamma_{\alpha \beta}^{0},
$$

and the divergence flux contribution $F^{i}$ is defined as

$$
\begin{aligned}
F^{i}= & \sqrt{-g}\left(\left(g^{0 j}-g^{00} V^{j}\right)\left(\left(P+P_{B}\right) \delta_{j}^{i}+Q_{j}^{i}\right)\right. \\
& \left.-\frac{1}{4 \pi}\left(B^{i} B^{0}-B^{0} B^{0} V^{i}\right)\right) .
\end{aligned}
$$

Ideally this total energy equation would be sufficient by itself. However, total energy schemes can run into trouble when recovering local values for the internal energy. Defining $\mathcal{E}_{D}$ as the non-thermal or "dynamical" component of the conserved energy,

$$
\mathcal{E}_{D}=\frac{D W}{\sqrt{-g}}+\frac{2 P_{B} W^{2}}{\sqrt{-g}}+\sqrt{-g}\left(g^{00} P_{B}-\frac{B^{0} B^{0}}{4 \pi}\right),
$$

we write

$$
\widetilde{E}=\frac{\left(\mathcal{E}-\mathcal{E}_{D}\right) \sqrt{-g} W}{\Gamma W^{2}+(\Gamma-1) g^{00}(\sqrt{-g})^{2}},
$$

for the internal energy extracted from the conserved energy field. The trouble arises when numerical truncation errors accumulate to the point that the sum of different physical contributions exceed the total energy $\left(\mathcal{E}_{D}>\mathcal{E}\right)$. This can occur in kinematic or magnetic field dominated flows and in the vicinity of strong shocks. The problem of negative energy can be avoided rather simply by forcing a minimum threshold on $\widetilde{E}$ to guarantee positivity. However, such a floor value is clearly not an accurate representation of the internal energy. Here we can benefit from having evolved the internal energy independently. We choose to only use the internal energy extracted from the total energy field whenever $\widetilde{E}>10^{-3} \mathcal{E}$. This avoids corrupting the internal energy value with numerical truncation error. The low density, background gas can also create accuracy problems for the total energy scheme because the density is occasionally reset to a numerical floor value. Therefore, we further require $\mathcal{E}>10^{-3} \mathcal{E}_{\max }$ as a condition for replacing $E$ with $\widetilde{E}$. This effectively excludes the background gas. Finally, to recover as much disk heating as possible we always use the larger of $E$ or $\widetilde{E}$, provided the above two conditions are met.

We find that the cooling timestep, $\Delta t_{\text {cool }}=c_{\mathrm{cff}} e / \Lambda$, is generally much shorter than the MHD timestep required for stability in the fluid evolution, $\Delta t_{\mathrm{MHD}}=c_{\mathrm{cfl}} \Delta x / V$, where $\Delta x$ and $V$ are characteristic zone lengths and velocities, respectively. Therefore, to save computational resources, we subcycle the cooling calculation, updating the energy $E$, as well as the temperature $T$ and scale height $H$, in each zone using only the cooling "source" term [final terms in equation (7)] and a timestep $\Delta t_{\text {cool }}$ until a full MHD timestep is reached, i.e. until $\sum_{i=1}^{N_{\text {steps }}}\left(\Delta t_{\text {cool }}\right)_{i}=\Delta t_{\mathrm{MHD}}$. Then we update the total energy $\mathcal{E}$ and momentum $S_{j}$ according to the final terms in equations (9) and (3), respectively. After that we proceed with the next MHD update for all other evolution terms using the normal timestep $\Delta t_{\mathrm{MHD}}$. Occasionally we have to deal with cooling timesteps that are unreasonably small due to very low temperatures or very high cooling efficiencies, regimes well outside the normal limits. To prevent the code from getting hung up at these points, we restrict $N_{\text {steps }}$ to be $\leq 100$. This limit is usually applied only in regions of very low density or very 
low energy where proper treatment of the fluid is inherently difficult.

\section{COOLING FUNCTION}

Three cooling processes are treated in this work: bremsstrahlung, synchrotron, and the inverse-Compton enhancement of each of these two. Generally, we implement the equations of Esin et al. (1996), with some changes. Below we describe first the equations that we use when the radiation is optically thin and some modifications that are necessary in order for the cooling computations to work well in our numerical simulations. We then describe the modifications necessary when the plasma becomes optically thick to these radiative processes. In the extremely optically thick limit, the treatment is essentially the diffusion approximation.

\subsection{Optically Thin Limit}

The total cooling rate for the optically thin gas is (Esin et al. 1996)

$$
q^{-}=\eta_{\mathrm{br}, \mathrm{C}} q_{\mathrm{br}}^{-}+\eta_{\mathrm{s}, \mathrm{C}} q_{\mathrm{s}}^{-},
$$

where $q_{\mathrm{br}}^{-}$and $q_{\mathrm{s}}^{-}$are the bremsstrahlung and synchrotron cooling terms, respectively, and $\eta_{\mathrm{br}, \mathrm{C}}$ and $\eta_{\mathrm{s}, \mathrm{C}}$ are Compton enhancement factors. The details of how to compute the Compton enhancements are given in Esin et al. (1996). Basically, $\eta(\nu)$ is a modified exponential function of the Compton parameter

$$
y=4\left(\Upsilon+4 \Upsilon^{2}\right)\left(\tau_{e s}+\tau_{e s}^{2}\right)
$$

where $\Upsilon \equiv k T_{e} / m_{e} c^{2}$ is the dimensionless electron temperature, and $\tau_{e s}$ is the electron scattering optical depth. $\eta(\nu)$ is limited to a maximum value of $3 k T / h \nu$, where $h$ is Planck's constant. $\eta_{\mathrm{br}, \mathrm{C}}$ is found implicitly by integrating $\eta(\nu) d q_{\mathrm{br}}^{-} / d \nu$ over appropriate frequencies, and $\eta_{\mathrm{s}, \mathrm{C}}$ is approximated as $\eta\left(\nu_{c}\right)$, where $\nu_{c}$ is the critical frequency below which the synchrotron emission becomes self-absorbed. Note: while we implement both enhancements, because synchrotron emission is dominant at temperatures where Comptonization becomes important, only $\eta_{\mathrm{s}, \mathrm{C}}$ is important in our simulations.

The un-Comptonized bremsstrahlung cooling rate from Esin et al. (1996) is

$$
q_{\mathrm{br}}^{-}=q_{e i}^{-}+q_{e e}^{-}+q_{ \pm}^{-}
$$

where

$$
\begin{aligned}
& q_{e i}^{-}=n_{p}\left(n_{e}+n_{+}\right) \times \\
& \left\{1.50 \times 10^{-22} \Upsilon^{0.5}\left(1+1.781 \Upsilon^{1.34}\right) \quad \Upsilon<1\right. \\
& \left\{2.12 \times 10^{-22} \Upsilon[\ln (1.123 \Upsilon+0.48)+1.5] \Upsilon \geq 1\right. \\
& q_{e e}^{-}=\left(n_{e}^{2}+n_{+}^{2}\right) \times \\
& \begin{cases}2.56 \times 10^{-22} \Upsilon^{1.5}\left(1+1.1 \Upsilon+\Upsilon^{2}-1.25 \Upsilon^{2.5}\right) \Upsilon<1 \\
3.42 \times 10^{-22} \Upsilon[\ln (1.123 \Upsilon)+1.28] & \Upsilon \geq 1\end{cases} \\
& q_{ \pm}^{-}=n_{e} n_{+} \times \\
& \begin{cases}3.43 \times 10^{-22}\left(\Upsilon^{0.5}+1.7 \Upsilon^{2}\right) & \Upsilon<1 \\
6.84 \times 10^{-22} \Upsilon[\ln (1.123 \Upsilon)+1.24] \Upsilon \geq 1\end{cases}
\end{aligned}
$$

in units of erg $\mathrm{cm}^{-3} \mathrm{~s}^{-1}$. These represent cooling due to electron-ion (17), positron-ion (17), electron-electron (18), positron-positron (18), and electron-positron 19 processes. Here $n_{p}=n_{e}-n_{+}$is the number density of protons and $n_{+}$is the number density of positronelectron pairs. One can determine the ratio $n_{+} / n_{p}=$ $\left(n_{+} / n_{e}\right) /\left(1-n_{+} / n_{e}\right)$ needed to calculate some of these terms using the following expression

$$
\begin{aligned}
& \frac{n_{+}}{n_{e}}=\frac{1}{\pi}\left\{1+\left[\frac{2 \Upsilon^{2}}{\ln (1.12 \Upsilon+1.3)}\right]\right\} \times \\
& \left\{\begin{array}{l}
2 \times 10^{-4} \Upsilon^{3 / 2} \exp (-2 / \Upsilon)(1+0.015 \Upsilon) \Upsilon \ll 1 \\
(112 / 27 \pi) \alpha_{f}^{2}(\ln \Upsilon)^{3}(1+0.058 / \Upsilon)^{-1} \Upsilon \gg 1,
\end{array}\right.
\end{aligned}
$$

where $\alpha_{f}$ is the fine structure constant.

The un-Comptonized synchrotron rate is a sum of optically thick and thin emission

$$
q_{s}^{-}=\frac{2 \pi k T}{H c^{2}} \int_{0}^{\nu_{c}} \nu^{2} d \nu+\int_{\nu_{c}}^{\infty} \epsilon_{s}(\nu) d \nu,
$$

where $H$ is the temperature scale height. The critical frequency can be found by equating the optically thin and thick volume emissivities at $\nu_{c}$

$$
\epsilon_{s}\left(\nu_{c}\right)=\frac{2 \pi}{H} \frac{\nu_{c}^{2}}{c^{2}} k T,
$$

and solving the above expression numerically. For an isotropic, full Maxwellian distribution of electrons and positrons, the optically thin volume emissivity is (Mahadevan et al. 1996; Esin et al. 1996)

$$
\begin{array}{r}
\epsilon_{s}(\nu, \vartheta)=4.43 \times 10^{-30} 4 \pi \nu\left(n_{e}+n_{+}\right) \times \\
\frac{I\left(x_{M} / \sin \vartheta\right)}{K_{2}(1 / \Upsilon)} \operatorname{ergs~cm}{ }^{-3} \mathrm{~s}^{-1}
\end{array}
$$

where $x_{M}=\nu / \nu_{M}$ is the normalized frequency (with $\nu_{M}=6.27 \times 10^{18} \mathrm{~B}(k T)^{2}[\mathrm{cgs}]$ being the critical electron frequency for a given temperature), $\vartheta$ is the angle between the observer and the magnetic field direction, and $K_{2}$ is the modified Bessel function of the second kind of order 2 , given by the integral

$$
K_{2}(1 / \Upsilon) \equiv \frac{\Upsilon^{2}}{3} \int_{1 / \Upsilon}^{\infty}\left(z^{2}-1 / \Upsilon^{2}\right)^{3 / 2} e^{-z} d z
$$

In the high-temperature limit, the electron-energyintegrated, unitless spectrum is given by the well-known expression (Pacholczyk 1970)

$$
I\left(\frac{x_{M}}{\sin \vartheta}\right) \equiv \frac{\sin \vartheta}{x_{M}} \int_{0}^{\infty} z^{2} e^{-z} F\left(x_{M} / z^{2} \sin \vartheta\right) d z
$$

where $F(x)$ is the normalized synchrotron spectrum for a single electron

$$
F(x)=x \int_{x}^{\infty} K_{5 / 3}(\xi) d \xi
$$

Esin et al. (1996) further average equation (23) over $\vartheta$ to obtain the total emissivity needed in equations 20 and 21

$$
\begin{gathered}
\epsilon_{s}(\nu)=4.43 \times 10^{-30} 4 \pi \nu\left(n_{e}+n_{+}\right) \times \\
\frac{I^{\prime}\left(x_{M}\right)}{K_{2}(1 / \Upsilon)} \operatorname{ergs~} \mathrm{cm}^{-3} \mathrm{~s}^{-1},
\end{gathered}
$$

The angle- and energy-integrated, unitless spectrum $I^{\prime}\left(x_{M}\right)$ can be fit to the following expression (Mahadevan 
et al. 1996)

$$
\begin{aligned}
I^{\prime}\left(x_{M}\right)= & \frac{4.0505}{x_{M}^{1 / 6}}\left(1+\frac{0.40}{x_{M}^{1 / 4}}+\frac{0.5316}{x_{M}^{1 / 2}}\right) \times \\
& \exp \left(-1.8899 x_{M}^{1 / 3}\right)
\end{aligned}
$$

with no more than $2.7 \%$ error over the range $0<x_{M}<$ $\infty$.

\subsection{Problems with the Cooling Functions}

Generally, equations [14-21) and (26-27) work fairly well in the temperature range $10^{8-\Pi 1} \mathrm{~K}$, which is the range over which they were used by Esin et al. (1996). However, this is not sufficiently broad for our numerical simulations where the plasma temporarily can attain very high or very low temperatures in a given cell. In applying these equations over the temperature range experienced in our simulations, we discovered the following problems:

1. The number of positrons and electrons (determined from equation 20 diverges for $T>2.4 \times 10^{11} \mathrm{~K}$, causing the simulation to crash.

2. There is an error in the synchrotron cooling expression (equation 26) that causes unphysical enhancement of the emission below $T<10^{8} \mathrm{~K}$. The error is so severe, that without a fix the simulations develop a cooling runaway, which freezes the plasma into a cold, toroidally-dominated magnetic state.

Our current fix for the first problem is very simple: we ignore positron cooling entirely (i.e., $n_{+}=0$ ). A complete fix to the positron/electron ratio calculation is being investigated at present. However, our cooled simulation generally remains below $10^{11} \mathrm{~K}$ in most places, so our neglecting the contribution of positron cooling is reasonably valid.

The second problem requires a more sophisticated solution. The error in the synchrotron cooling is caused by the use of different lower integration limits in equations (23) and (24). $K_{2}(1 / \Upsilon)$ is the correct factor only if lower temperatures $(\Upsilon \rightarrow 0)$ are allowed in equation (24) also. The result of this limit mismatch is that the denominator in equation (26) vanishes faster for low temperatures $\left(T<10^{8} \mathrm{~K}\right)$ than the numerator, leading to enormous cooling rates for plausible temperatures (in the range $\left.10^{5-7} \mathrm{~K}\right)$. Such a problem would not have affected Esin et al. (1996)'s results, which maintained temperatures above this range. However, in a numerical simulation with many millions of cooling computations over millions of cells and time steps, the probability is quite high that such cool temperatures will be attained somewhere in the flow, whereupon the entire structure will catastrophically freeze.

Since we still wish to use equation (27) for $I^{\prime}\left(x_{M}\right)$, the best fix for this problem is simply to assume the same high temperature limit in equation (23) as was assumed in equation (24) (i.e., allow $1 / \Upsilon \rightarrow 0$, which is equivalent to replacing $\left.K_{2}(1 / \Upsilon) \rightarrow 2 \Upsilon^{2}\right)$, resulting in a corrected synchrotron emissivity expression

$$
\begin{aligned}
\epsilon_{s}(\nu)= & 4.43 \times 10^{-30} 2 \pi \nu\left(n_{e}+n_{+}\right) \times \\
& \frac{I^{\prime}\left(x_{M}\right)}{\Upsilon^{2}} \operatorname{ergs~} \mathrm{cm}^{-3} \mathrm{~s}^{-1},
\end{aligned}
$$

to be used instead of equation (26) in equation 20. The error introduced by using the high-temperature limit of $K_{2}$ is of the same order as that caused by using a zero lower limit in the numerator of equation (24) (i.e., $\left.I\left(x_{M} / \sin \vartheta\right)\right)$ in the first place. And that error is negligible compared to the total plasma emission, because it occurs at low temperatures where bremsstrahlung dominates (see Fig. 1).

Finally, a Saha ionization equation is used to determine the electron density, which provides an exponential cutoff in the cooling below $T \sim 10^{4} \mathrm{~K}$. Thus, we consider continuum cooling only; no line or molecular cooling is included.

One cautionary comment about these cooling functions is worth noting. The expressions contain many exponentials whose arguments easily can trigger underflow or overflow in a digital computer. If great care is not taken in respecting these limitations, even correct coding of the cooling functions will lead to non-physical results (heating or cooling runaways) in the accretion flow.

\subsection{Optically Thick Limit}

To represent the cooling behavior in the optically thick limit, we use a slightly modified version of Esin et al. (1996)'s equation (21) that is suitable for multidimensional MHD simulations. Our total cooling function is given by Hubeny (1990)

$$
\begin{aligned}
\Lambda & =\frac{-q^{-}}{1+\sqrt{3} \tau_{\mathrm{abs}}+\frac{3}{2} \tau \tau_{\mathrm{abs}}} \\
& =\frac{4 \sigma T^{4} / H}{\frac{3}{2} \tau+\sqrt{3}+1 / \tau_{\mathrm{abs}}},
\end{aligned}
$$

where the local temperature scale height is computed from

$$
H=\frac{T^{4}}{\left|\nabla\left(T^{4}\right)\right|} .
$$

This is a suitable definition for scale height in a multidimensional numerical simulation. If the flow were to assume a thin disk structure, for example, equation 30 would give the standard exponential (radiation energy density) scale height.

The optical depth due to absorption is calculated as

$$
\tau_{\mathrm{abs}}=\kappa_{\mathrm{abs}} \rho H
$$

with

$$
\kappa_{\mathrm{abs}}=\frac{q^{-}}{4 \sigma T^{4} \rho} .
$$

And the total optical depth is computed using an averaged opacity

$$
\tau=\langle\kappa\rangle \rho H
$$

with the diffusion average of $\kappa$ being

$$
\begin{aligned}
\langle\kappa\rangle & \equiv \frac{-2\left|\nabla\left(T^{4}\right)\right|^{2}}{\rho T^{4} \nabla \cdot\left[\nabla\left(T^{4}\right) /(\kappa \rho)\right]} \\
& =\frac{-2 T^{4}}{\rho H^{2} \nabla \cdot\left[\nabla\left(T^{4}\right) /(\kappa \rho)\right]} .
\end{aligned}
$$

The total opacity used in this equation is given by $\kappa=\kappa_{\text {abs }}+\kappa_{\text {es }}$ with $\kappa_{\text {es }}=0.4 \mathrm{~cm}^{2} \mathrm{~g}^{-1}$ being the electron scattering opacity. Note that our definitions for $H$ and 


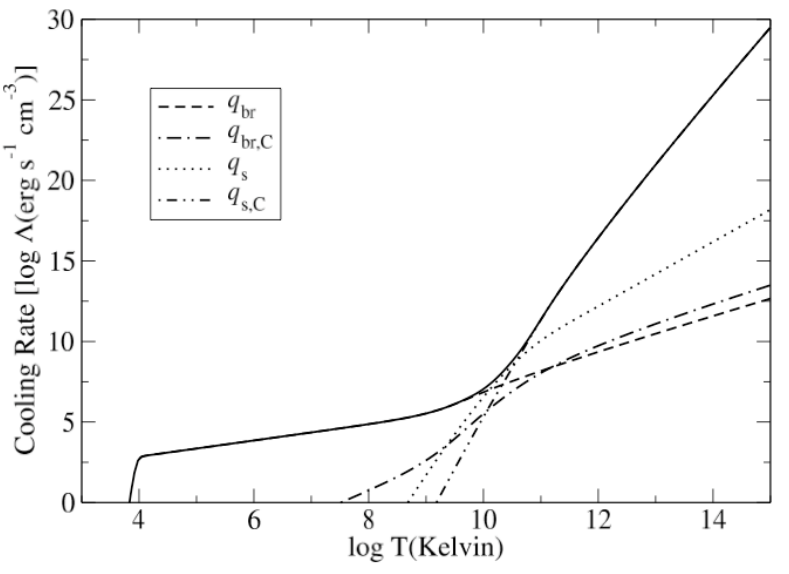

Fig. 1.- Plot of a sample cooling function with $\rho=10^{-10} \mathrm{~g}$ $\mathrm{cm}^{-3}, B=8380 \mathrm{G}$, and $H=2.7 \times 10^{7} \mathrm{~cm}$. The total cooling function (solid line) and the following components are represented: bremsstrahlung (short-dashed); Compton enhancement to bremsstrahlung (dot-long dash); synchrotron (dotted); and the Compton enhancement to synchrotron (dot-dot-dash).

$\langle\kappa\rangle$ allow for redistribution of heat within an optically thick region $(\tau>>1)$; equation $(29)$ then reduces exactly to the diffusion approximation. Our approach also allows for non-local heating outside a marginally thickthin transition region $(\tau \approx 1)$ by photons from more optically thick regions, since the partially diffusive nature of equation 35 in this situation allows partial transport of heat from warmer to cooler regions. However, in the optically thin case $\left(\tau<<1\right.$ and $\left.\Lambda=q^{-}\right)$, there is no transport of heat from one region of the simulation to the other. There is only heat loss from the plasma and, therefore, from the simulation grid.

For the purpose of illustration, in Figure 1 , we plot the total cooling function $\Lambda$ as a function of temperature, assuming fixed values of $\rho, H$, and $B$. Of particular note is the temperature range over which each of the cooling processes is important. For $10^{4} \lesssim T \lesssim 10^{9} \mathrm{~K}$, the dominant process is bremsstrahlung. At higher temperatures bremsstrahlung no longer dominates, even though its dependence with temperature steepens to be proportional to $T \log T$. Instead, above $10^{9} \mathrm{~K}$ the dominant cooling process is synchrotron, with Compton enhancement of synchrotron becoming important for temperatures a little above that. Comptonization of bremsstrahlung, while included, is never particularly important in our simulations.

\section{INITIALIZATION}

We initialize these simulations starting from the analytic solution for a constant specific angular momentum torus around a non-rotating black hole (Kozlowski et al. 1978). In our initialization, the torus is defined by its inner radius $r_{\text {in }}=150 r_{G}$ and the radius of the pressure maximum $r_{\text {center }}=200 r_{G}$. Knowledge of $r_{\text {center }}$ leads directly to a determination of $\ell$, the specific angular momentum of the torus, by setting it equal to the geodesic value at that radius. Having chosen $r_{\text {in }}$ we can obtain $u_{\text {in }}=u_{t}\left(r_{\text {in }}\right)$, the surface binding energy of the torus, from $u_{t}^{-2}=g^{t t}+\ell^{2} g^{\phi \phi}$.

The solution of the torus variables can now be specified. The internal energy of the torus is (Hawley et al.
1984)

$$
\epsilon(r, \theta)=\frac{1}{\Gamma}\left[\frac{u_{i n}}{u_{t}(r, \theta)}\right] .
$$

Thus the initial temperature of the torus, $T_{0}=(\Gamma-$ 1) $\left(\mu m_{H} / k\right) \epsilon$, is fixed to be $\approx 10^{9} \mathrm{~K}$ by the specification of the torus. Assuming an isentropic equation of state $P=\rho \epsilon(\Gamma-1)=\kappa \rho^{\Gamma}$ for the initialization, the density is given by $\rho=[\epsilon(\Gamma-1) / \kappa]^{1 /(\Gamma-1)}$. We take $\Gamma=5 / 3$ and $\kappa=5 \times 10^{22}$ (cgs units). This gives an initial density maximum in the torus of $\rho_{\text {max }, 0}=2.8 \times 10^{-9} \mathrm{~g} \mathrm{~cm}^{-3}$. Finally, the angular velocity of the fluid is specified by

$$
\Omega=V^{\phi}=-\ell \frac{g_{t t}}{g_{\phi \phi}} .
$$

Once the torus is constructed, it is seeded with a weak dipole magnetic field in the form of poloidal loops along the isobaric contours within the torus. The initial magnetic field vector potential is (De Villiers \& Hawley 2003)

$$
A_{\phi}=\left\{\begin{array}{cl}
b\left(\rho-\rho_{\text {cut }}\right) & \text { for } \rho \geq \rho_{\text {cut }} \\
0 & \text { for } \rho<\rho_{\text {cut }}
\end{array} .\right.
$$

The non-zero spatial magnetic field components are then $\mathcal{B}^{r}=-\partial_{\theta} A_{\phi}$ and $\mathcal{B}^{\theta}=\partial_{r} A_{\phi}$. The parameter $\rho_{\text {cut }}=$ $0.5 * \rho_{\max , 0}$ is used to keep the field a suitable distance inside the surface of the torus. Using the constant $b$ in equation (38), the field is normalized such that initially $\beta=P / P_{B} \geq \beta_{0}=10$ throughout the torus. The choice of the initial field geometry has been shown to have relatively little effect on the development of the MRI and the evolution of the disk (Beckwith et al. 2008), which is all we are focused on in this manuscript. However, the initial field topology does imprint itself in the formation and evolution of jets, meaning that we will need to perform a more widely varying set of simulations before addressing that topic.

In the background region not specified by the torus solution, we set up a static, low density $\left(\rho=10^{-6} \rho_{\max , 0}\right)$, non-magnetic, hot gas. Numerical floors are placed on $\rho$ and $e$ at approximately $10^{-12}$ and $10^{-10}$ of their initial maxima, respectively. The density floor is very seldom applied once the initial background is replaced by evolved disk material. The energy floor is applied somewhat more frequently. Nevertheless, these very low floor values should not have any significant dynamical impact on the problem.

These simulations are performed in 2.5 spatial dimensions (all three spatial components of vector quantities are evolved, although symmetry is assumed in the azimuthal direction) using a spherical polar coordinate grid. The grid used in the majority of the simulations consists of 192 radial zones and 128 zones in $\theta$. We also performed select simulations at one-half and at double this resolution to test the numerical convergence of our results. We find very little variation between our default resolution and the higher resolution simulation, suggesting our results are well converged.

In the radial direction we use a logarithmic coordinate of the form $\eta \equiv 1.0+\ln \left(r / r_{\mathrm{BH}}\right)$. The spatial resolution near the black hole horizon is $\Delta r \approx 0.05 r_{G}$; near the initial pressure maximum of the torus, the resolution is $\Delta r \approx 5 r_{G}$. Both are considerably smaller than the initial 
TABLE 1

MODELS

\begin{tabular}{cc}
\hline \hline Name & Description \\
\hline I & Internal-energy evolving \\
I+C & internal energy + cooling \\
T & Total-energy conserving \\
T+C & Total energy + cooling
\end{tabular}

characteristic MRI wavelength $\lambda_{\mathrm{MRI}} \equiv 2 \pi v_{\mathrm{A}} / \Omega \approx 50 r_{G}$. In the angular direction, we use a concentrated latitude coordinate $x_{2}$ of the form $\theta=x_{2}+\frac{1}{2}(1-h) \sin \left(2 x_{2}\right)$ with $h=0.5$, which concentrates resolution toward the midplane of the disk. As a result $r_{\text {center }} \Delta \theta=4 r_{G}$ near the midplane while it is a factor of $\sim 3$ larger for the zones near the pole.

For this work we have run the Cosmos ++ numerical code in four different modes: 1) internal-energy evolving with no explicit cooling (model 522I or simply I); 2) internal-energy evolving including an explicit cooling function (model 522IC or simply I+C); 3) total-energy conserving with no explicit cooling (model $522 \mathrm{~T}$ or simply T); and 4) total-energy conserving including an explicit cooling function (models 522TC or simply $\mathrm{T}+\mathrm{C}$ ). The motivation for this is to allow for a clear, direct comparison of simulations carried out under different physical assumptions. The " 522 " in the long naming convention is a reference to our choice of $\kappa=5 \times 10^{22}$.

\section{RESULTS}

Since no cooling processes are treated in simulations I and $\mathrm{T}$, those results simply scale with the mass of the black hole. However, for purposes of comparison with simulations $\mathrm{I}+\mathrm{C}$ and $\mathrm{T}+\mathrm{C}$, we will assume the same scale for all variables in each simulation. Specifically we assume a black hole mass of $M=10 M_{\odot}$, which sets the following physical scales in the initial torus: $r_{\text {in }}=2.2 \times 10^{8}$ $\mathrm{cm}$ and $r_{\text {center }}=3.0 \times 10^{8} \mathrm{~cm}$. The orbital period at $r=r_{\text {center }}$ is $t_{\text {orb }}=1.77 \times 10^{4} M=0.875 \mathrm{~s}$. The initial gas densities and temperatures are $\rho_{\max , 0}=2.8 \times 10^{-9}$ $\mathrm{g} \mathrm{cm}^{-3}, \rho_{\text {background }}=2.8 \times 10^{-15} \mathrm{~g} \mathrm{~cm}^{-3}, T_{\text {disk }} \approx 10^{9}$ $\mathrm{K}$, and $T_{\text {background }} \approx 10^{11} \mathrm{~K}$. The mass accretion rate is scaled by the Eddington rate $\dot{M}_{\text {Edd }}=8.4 \times 10^{18} \mathrm{~g} \mathrm{~s}^{-1}$ for an $M=10 M_{\odot}$ black hole. The models and parameters are summarized in Tables 1 and 2. Each simulation is evolved for seven orbital periods. This is sufficient time for all four models to achieve approximate equilibriums inside $r \approx r_{\text {in }}=150 r_{G}$. However, because these simulations are carried out in two dimensions, the antidynamo theorem prevents a true steady-state from being achieved, so these are only approximations of the true state.

\subsection{Internal-Energy Evolving with No Cooling}

In this simulation, which we designate 522I or just I, we only evolve the internal energy equation (equation 7), ignoring the cooling function $(\Lambda=0)$. This mode of evolution has commonly been used in the past (e.g., De Villiers \& Hawley 2003, Anninos et al. 2005), particularly in codes derived from the pioneering work of Wilson (1972). From a thermodynamics perspective, running the code in this mode is an interesting case study. Because total energy is not conserved, any kinetic or magnetic energy
TABLE 2

PARAMETERS

\begin{tabular}{cc}
\hline \hline Name & Initial value \\
\hline$r_{\text {in }}$ & $150 r_{G}=2.2 \times 10^{8} \mathrm{~cm}$ \\
$r_{\text {center }}$ & $200 r_{G}=3.0 \times 10^{8} \mathrm{~cm}$ \\
$t_{\text {orb }}$ & $1.77 \times 10^{4} M=0.875 \mathrm{~s}$ \\
$\rho_{\text {max }, 0}$ & $2.8 \times 10^{-9} \mathrm{~g} \mathrm{~cm}^{-3}$ \\
$\rho_{\text {background }}$ & $2.8 \times 10^{-15} \mathrm{~g} \mathrm{~cm}^{-3}$ \\
$T_{\text {disk }}$ & $\approx 10^{9} \mathrm{~K}$ \\
$T_{\text {background }}$ & $\approx 10^{11} \mathrm{~K}$ \\
$M_{B H}$ & $10 M_{\odot}$ \\
$\dot{M}_{\text {Edd }}$ & $8.4 \times 10^{18} \mathrm{~g} \mathrm{~s}^{-1}$ \\
& \\
\hline
\end{tabular}

dissipated in the disk (except through shocks) is simply lost from the simulation. In a sense, though, this creates a sort of thermodynamic equilibrium, wherein cooling (in the sense of energy lost from the disk) exactly matches dissipative heating everywhere in the simulation. Thus, without explicitly treating heating or cooling, this simulation actually mimics one that includes heating plus a highly efficient cooling process. Some caution is in order, though, in making such a statement. Some heating and cooling mechanisms are captured in equation (7), specifically shock heating (through the artificial viscosity term) and adiabatic heating and cooling. These may not be balanced in the same way they would in a simulation that rigorously treated both heating and cooling. Furthermore, this treatment implies rapid, efficient cooling throughout the computational domain, regardless of physical conditions. We will explore this point further in $\$ 5.4$. In the upper-left panel of Figure 2, we plot the final distribution of gas density and temperature for this model.

\subsection{Internal-Energy Evolving with Cooling}

For this simulation, which we designate 522IC or simply $\mathrm{I}+\mathrm{C}$, we again evolve the internal-energy equation (equation 7), this time including the radiative cooling term. Physically speaking, there is relatively little motivation for this model, as we know there are important dissipative heating processes in disks that are ignored in this model. Nevertheless, this model does serve to round out our small lattice of tests and demonstrate the importance of using a fully conservative energy scheme (or some other heat-capturing procedure) when including radiative cooling processes. This is because, without including heating, there is nothing to counterbalance the cooling, and the disk ends up unreasonably cold and thin, with temperatures below $10^{7} \mathrm{~K}$ over much of the disk midplane, as shown in the upper-right panel of Figure2,

\subsection{Total-Energy Conservation with No Cooling}

For this simulation, which we designate $522 \mathrm{~T}$ or simply $\mathrm{T}$, we use the total energy conserving mode of Cosmos++ (again with $\Lambda=0$ ). By evolving equation (9) and conserving total energy, we effectively capture dissipative heating mechanisms ignored in the previous simulations since any losses to the kinetic or magnetic energy of the gas are recovered as heat. Total energy conserving codes (as used previously by e.g., McKinney 2006; Noble et al. 2007), are particularly applicable when considering radiatively inefficient accretion flows (RIAFs), such 


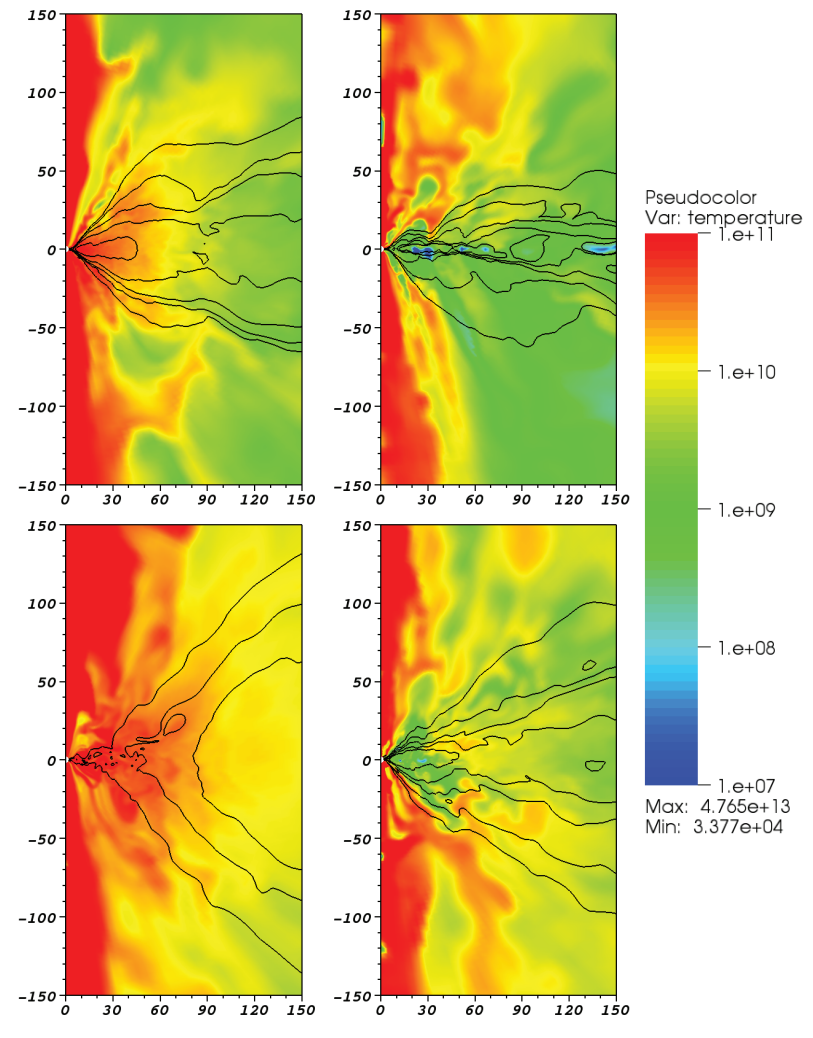

FIG. 2.- Pseudo-color plots of $\log (T)$ with contours of $\log \rho$. The upper-left panel is the final time dump of the internal energy model I; the upper-right panel is the final time dump of the internal energy plus cooling model I+C; the lower-left panel is the final time dump of the total energy model $\mathrm{T}$; and the lower-right panel is the final time dump of the total energy plus cooling model $\mathrm{T}+\mathrm{C}$. The density contours are at $\rho=0.005,0.016,0.05,0.16$, and $0.5 \rho_{\max , 0}$.

as the one that is thought to be currently feeding Sgr A* (Narayan et al. 1995: Yuan et al. 2003). Because these disks (both simulated and real) are not able to radiate their heat away efficiently, they tend to be very hot and vertically thickened, as shown in the lower-left panel of Figure 2

\subsection{Total Energy Conservation with Cooling}

For this simulation, which we designate $522 \mathrm{TC}$ or $\mathrm{T}+\mathrm{C}$, we use the dual energy evolving mode of Cosmos ++ described in $\$ 2$. By including equation $(9)$ and conserving total energy, we again effectively capture dissipative heating mechanisms in the disk. In addition to the total energy equation, we simultaneously evolve the internal energy equation (7) to ensure we recover reasonable values for the internal energy whenever the total energy budget is dominated by non-thermal components. This is particularly important for calculating the temperature of the gas, which is a crucial input into the cooling routine. This is the only simulation where the dissipative heating processes are balanced by a physically motivated local cooling function, as described in 93 . As expected, this leads to an intermediate disk state between the hot, thickened RIAF state of simulation $\mathrm{T}$ and the unrealistically cooled disk in simulation $\mathrm{I}+\mathrm{C}$. The results are shown in the lower-right panel of Figure 2 ,

\section{COMPARISON OF NUMERICAL MODELS}

Simply looking at Figure 2 and comparing the four models, we already note a number of qualitative differences. Obviously the disk in model T, which is expected to capture heating appropriately but includes only adiabatic cooling, is much hotter and thicker than any of the other three simulations. This is consistent with the expectations of a radiatively inefficient, two-temperature gas, where the ions are poorly coupled to the electrons. The opposite extreme is model $\mathrm{I}+\mathrm{C}$, which includes radiative cooling processes without capturing most of the real, physical heating in the disk. This leads to a very thin, cold disk solution, which could only apply in cases of very weak turbulence or very low ionization.

More interesting is to compare models I, the internalenergy evolving model, and $\mathrm{T}+\mathrm{C}$, the total energy plus cooling model. As we said before, model I can be thought of as a radiatively efficient model, but an unphysical one where cooling equals heating practically everywhere in the flow. This gives a much cooler disk than in model T, but also one in which the temperature increases monotonically as gas moves radially inward through the disk (compressive heating becomes more important). This is in contrast to model $\mathrm{T}+\mathrm{C}$, which shows an approximately constant or even slightly decreasing temperature for $r<150 r_{G}$, due to the efficiency of Compton enhanced synchrotron radiation. Next we make a more quantitative comparison of the models.

\subsection{Angle-Averaged Properties of the Simulations}

First, we construct density-weighted spherical shell averages of the various disk properties. The formula we use is

$$
\langle\mathcal{Q}\rangle_{A}(r, t)=\frac{1}{A} \int_{0}^{2 \pi} \int_{0}^{\pi} \mathcal{Q} \sqrt{-g} \mathrm{~d} \theta \mathrm{d} \phi,
$$

where $A=\int_{0}^{2 \pi} \int_{0}^{\pi} \sqrt{-g} \mathrm{~d} \theta \mathrm{d} \phi$ is the surface area of the shell. We also average these quantities over the final two orbital periods of the simulations, $5 t_{\text {orb }}=t_{\min } \leq t \leq$ $t_{\max }=7 t_{\text {orb }}$, to negate any transient features. The time averages are defined as

$$
\langle\mathcal{Q}\rangle_{t}=\frac{1}{t_{\max }-t_{\min }} \int_{t_{\min }}^{t_{\max }} \mathcal{Q} \mathrm{d} t
$$

The numerical results for the internal-energy model I, the internal-energy plus cooling model $\mathrm{I}+\mathrm{C}$, the totalenergy model $\mathrm{T}$, and the total-energy plus cooling model $\mathrm{T}+\mathrm{C}$ are shown in Figure 3. We have also included the predictions for the transition state solution, which we discuss below (Section 7.1).

There are clear outliers among the various disk models. For instance, the total-energy conserving model $\mathrm{T}$ exhibits significantly lower density, pressure, and azimuthal magnetic field in the inner regions than any of the other three simulations. This actually owes to its much lower accretion rate (shown in Figure 5); material is just not moving through the disk very quickly. This, coupled with the considerably larger thickness of model $\mathrm{T}$, leads to very low density and pressure. The internal-energy plus cooling model $\mathrm{I}+\mathrm{C}$ is an outlier in the other direction, being an order of magnitude cooler and thinner than model $\mathrm{T}$. Models I and $\mathrm{T}+\mathrm{C}$, on the other hand, representing unphysical and physical cooling, respectively, look very 

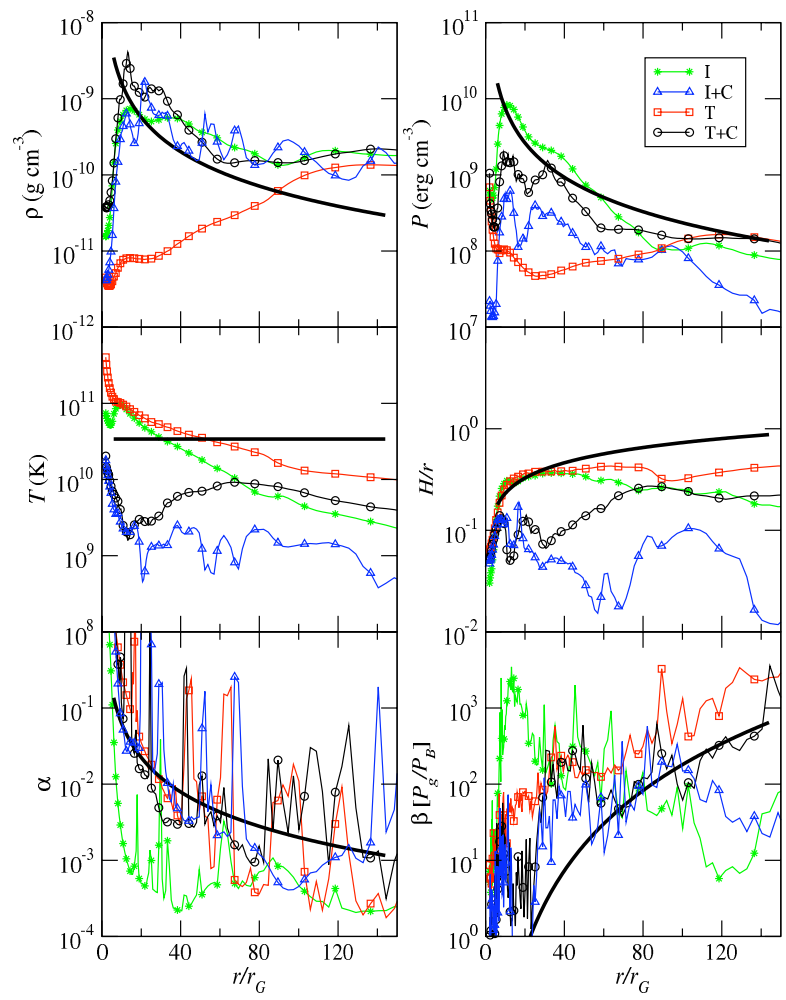

FIG. 3.- Main disk properties plotted as a function of radius for the internal-energy model I, the internal-energy plus cooling model $\mathrm{I}+\mathrm{C}$, the total-energy model $\mathrm{T}$, and the total-energy plus cooling model $\mathrm{T}+\mathrm{C}$. The data have been time-averaged over the final two orbital periods of each simulation. $P, T, \alpha$, and $\beta$ are density-weighted averages. The thick solid line in each frame is the solution for the MDAF transition region from equations 43.

similar in many regards. In fact, the only notable exceptions are in $T, \alpha$, and $\beta$. We mentioned the difference in $T$ above, which is owing to the efficiency of Comptonenhanced synchrotron cooling for $T \gtrsim 10^{10} \mathrm{~K}$, and will return to the differences in $\alpha$ and $\beta$ below.

\subsection{Volume-Integrated Properties of the Simulations}

In Figure 6 we plot the total integrated internal and magnetic energies for each of our four classes of models. In the magnetic energy we can see the characteristic growth of the magneto-rotational instability on an orbital timescale, after which it saturates. After about 2-3 orbital periods the magnetic energy begins to decay due to accretion into the black hole, advection off of the grid through the action of jets and winds, and also due to the Cowling anti-dynamo theorem (the magnetic field is not able to regenerate itself in two dimensions).

Initially there is very little heating in the disk as the magneto-rotational instability has not had time to build up the turbulence and the flow is mostly laminar. Thus, in models I (which fails to capture heating realistically), $\mathrm{I}+\mathrm{C}$ (which neglects heating, yet includes cooling), and $\mathrm{T}+\mathrm{C}$ (which captures heating, yet also includes cooling), the disk initially begins to cool. Once the MRI really kicks in after about 1 orbit, models I, T, and $\mathrm{T}+\mathrm{C}$ begin heating, although model I heats more slowly than the two total energy conserving models. Model $\mathrm{I}+\mathrm{C}$ never shows significant heating, clearly demonstrating that the
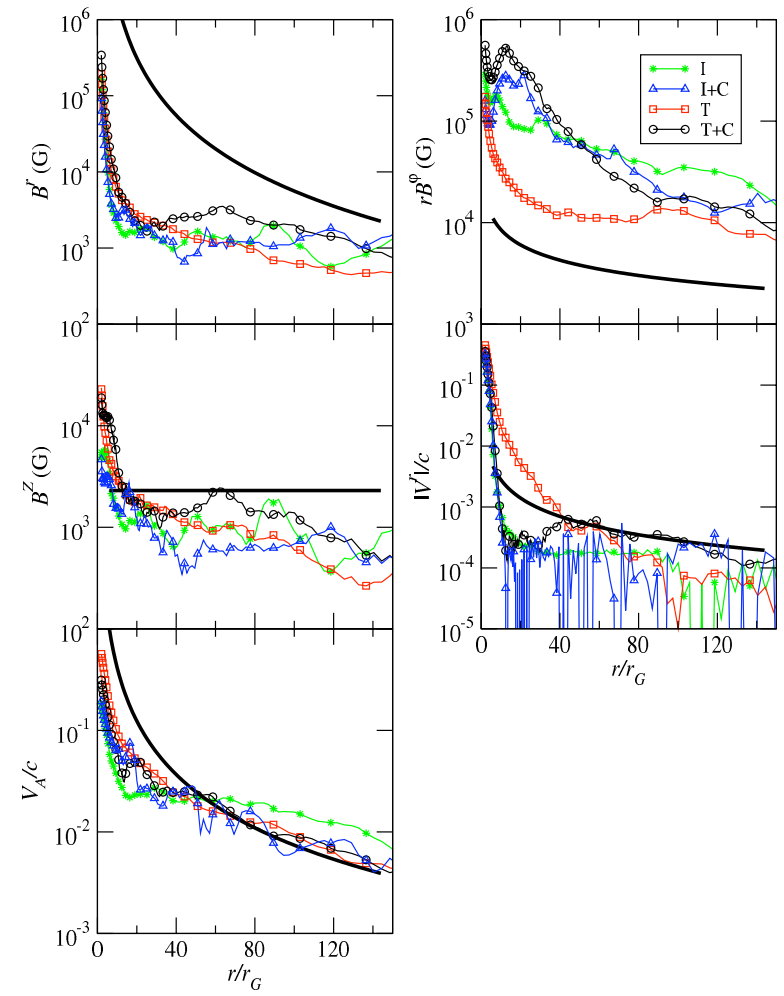

FIG. 4.- Fig. 3 continued. $B^{r}, B^{\phi}, B^{Z}, V^{r}$, and $V_{A}$ are densityweighted averages.

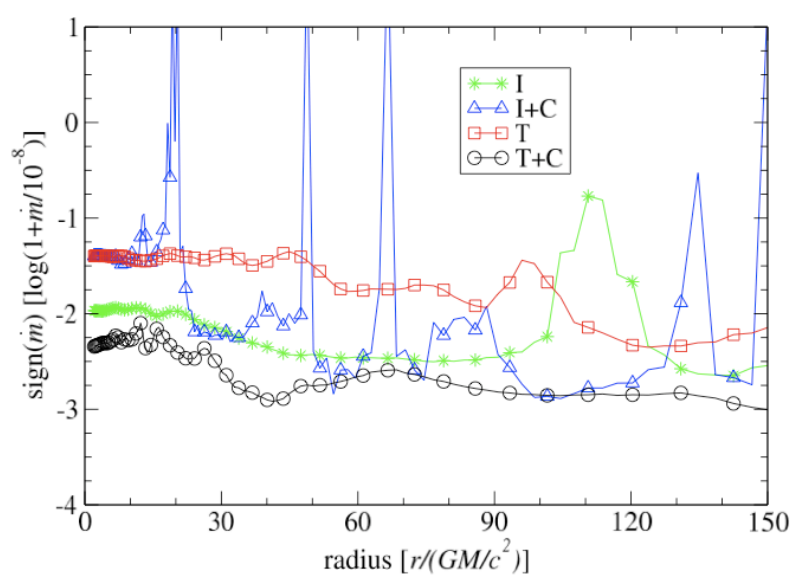

FIG. 5.- Plot of $\operatorname{sign}(\dot{m}) \log \left(1+\dot{m} / 10^{-8}\right)$ as a function of radius for the internal-energy model I, the internal-energy plus cooling model $\mathrm{I}+\mathrm{C}$, the total-energy model $\mathrm{T}$, and the total-energy plus cooling model $\mathrm{T}+\mathrm{C}$, where $\dot{m}=\dot{M} / \dot{M}_{\mathrm{Edd}}$. The data have been time-averaged over the final two orbital periods of each simulation. By this time all three simulations have achieved a reasonably steady inflow solution for $r \lesssim 150 r_{G}$.

local cooling always dominates. Once heating begins, the non-radiative models I and $\mathrm{T}$ never stop heating, whereas cooling appears to catch up with heating in the radiatively-cooled total-energy model $\mathrm{T}+\mathrm{C}$ after about 4 orbits.

The differences between the internal energy curves of models $\mathrm{I}$ and $\mathrm{T}$ in Figure 6 give some indication of the 


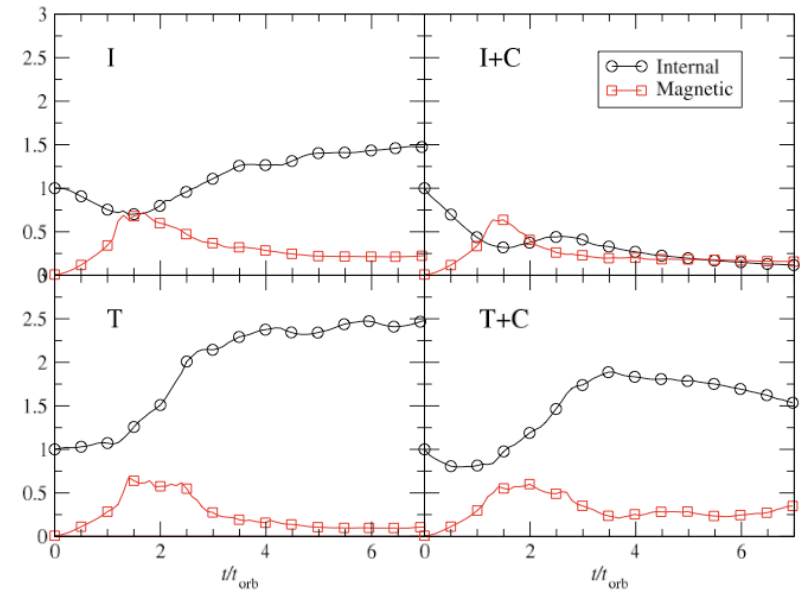

FIG. 6.- Plots of total internal (circular symbols) and magnetic (square symbols) energies as functions of time for the internalenergy model I (top-left panel), the internal-energy plus cooling model I+C (top-right panel), the total-energy model $\mathrm{T}$ (bottomleft panel), and the total-energy plus cooling model $\mathrm{T}+\mathrm{C}$ (bottomright panel). The energy scales have been normalized to the initial internal energy in the simulations.

amount of energy simply lost from the simulation by model I, which uses only the internal energy formulation. This amount of energy is comparable to the amount of internal energy initially contained in the simulation. Likewise, the difference between the internal energy curves of models $\mathrm{T}$ and $\mathrm{T}+\mathrm{C}$ say something about the level of cooling in the disk. The physical cooling in model $\mathrm{T}+\mathrm{C}$ is of the same magnitude as the unphysical cooling of model I, but as we have already shown, the resulting disk structure has significant quantifiable differences.

We note here that the internal energy in the lowerleft panel of Figure 6 (model T) continues to increase all the way to the end of the simulation. This suggests that dissipative heating of the disk has not yet been fully quenched by anti-dynamo processes, so the cooling seen in model $\mathrm{T}+\mathrm{C}$ is genuine.

\section{COMPARISON OF NUMERICAL AND ANALYTIC RESULTS}

Ultimately we would like to make direct comparisons between our numerical results and observations of blackhole accretion disks in the Hard state. In the meantime we can also compare our numerical results with applicable analytic work. An interesting comparison can be made between our radiatively-cooled model $\mathrm{T}+\mathrm{C}$ and the MDAF model. The basic idea of the MDAF is that catastrophic cooling in the inner region of the disk should cause the disk to collapse vertically and dramatically reduce the thermal energy relative to the magnetic, such that $\beta=P_{\text {gas }} / P_{B}$ becomes $<1$ (Meier 2005, 2008). It is clear from Figure 2 that the radiatively cooled disk $(\mathrm{T}+\mathrm{C})$ indeed is much thinner than the uncooled disk (T). However, it is apparent from Figure 7, where we plot $\beta$ over much of the domain of the simulation that although $\beta$ is significantly lower in some regions of the radiatively cooled disk relative to the uncooled disk (particularly for $r<100 r_{G}$ ), its angle average is not less than unity at any radius.

Apparently we did not achieve a fully magnetically dominated state. Nevertheless, we can make a quantita-
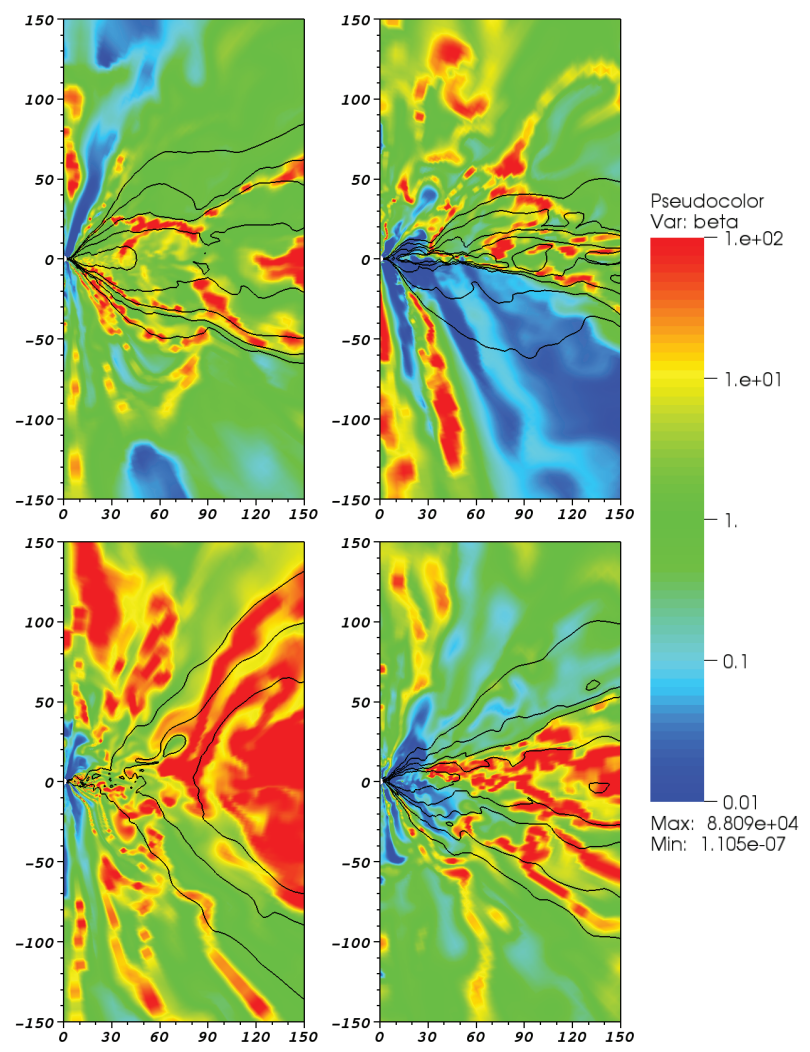

FIG. 7. - Pseudo-color plots of $\beta$ with contours of $\log \rho$. Panel $a$ is the final time dump of the internal-energy model I; Panel $b$ is the final time dump of the internal-energy plus cooling model $\mathrm{I}+\mathrm{C}$; Panel $c$ is the final time dump of the total-energy model T; and Panel $d$ is the final time dump of the total-energy plus cooling model $\mathrm{T}+\mathrm{C}$. The density contours are as in Fig. 2

tive comparison between our numerical model $\mathrm{T}+\mathrm{C}$ and the predictions of the MDAF model. Prior to becoming magnetically dominated, the model predicts that the inflow should pass through a "transitional" state, in which $\beta$ decreases from its initially large value to of order unity. It is this transitional inflow solution, then, that we wish to compare with our numerical simulation.

\subsection{Analytic Theory of Transitional Flow}

Analytic development of MDAF theory begins with the development of a simple analytic model for the ADAF structure in the region where the ion and electron temperatures are definitely equal (i.e., $r \gtrsim 144 r_{G}$ ). The simple ADAF model is constructed in a manner similar to the Shakura \& Sunyaev (1973) $\alpha$-model, except that cooling of the How is performed by an advective term $\left[Q_{a d v} \approx \dot{M} P /\left(2 \pi r^{2} \rho\right)\right]$ instead of the usual radiative term. The Compton parameter in this optically thin flow

$$
y \approx 16 \Upsilon^{2} \tau_{e s}
$$

remains less than unity for $r>R_{0}$, where

$$
R_{0}=2.75 \times 10^{8} \alpha^{-2 / 5} \mathrm{~mm}^{2 / 5} \mathrm{~cm} .
$$

is defined as the radius where $y=1$ in the ADAF, with $m=M / M_{\odot}$, and $\dot{m}=\dot{M} / \dot{M}_{\text {Edd }}$. Compton cooling is unimportant until the inflow approaches this radius. Note that the electron scattering optical depth 
$\tau_{e s}=\kappa_{e s} \rho H$ uses the electron scattering opacity $\kappa_{e s}$ and disk scale height $H$. Because we use spherical geometry, the scale height is equivalent to

$$
H \equiv r \sin \Theta
$$

where $\Theta$ is the disk angular scale height, with most of the accretion flow occurring in a polar angle range of $\pi / 2-\Theta<\theta<\pi / 2+\Theta$.

Inside $R_{0}, y$ must be $\geq 1$, Compton cooling becomes important, and the transitional flow begins. In fact, the analytic MDAF models assume that generic Compton cooling is dominant, so $y \approx 1$ must be true for $r<R_{0}$ (Shapiro et al. 1976). This cooling decreases the plasma temperature in the transition region, and therefore the disk scale height, which also enhances $B^{r}$ and $r B^{\phi}$ by compression. Conservation of magnetic flux in the steady MHD inflow requires that

$$
\begin{aligned}
B^{r} & \propto r^{-1} H^{-1} \\
r B^{\phi} & \propto\left(V^{r}\right)^{-1} H^{-1} \\
B^{Z} & \propto\left(V^{r}\right)^{-1} r^{-1}
\end{aligned}
$$

This means that the magnetic viscosity stress parameter $\alpha$ no longer can remain uniform with radius

$$
\alpha(r)=\frac{t^{\phi r}}{P} \propto \frac{r B^{\phi} B^{r}}{P} \propto\left(\frac{r}{H}\right)^{3}
$$

Since $H \propto r^{3 / 2}$ in this solution, the magnetic stress relative to the pressure must increase as the inflow approaches the black hole $\left(\alpha \propto r^{-3 / 2}\right)$. The end of transitional flow, and beginning of true MDAF flow, begins inside the radius

$$
R_{1}=2.75 \times 10^{8} \alpha^{4 / 15} \mathrm{~mm}^{2 / 5} \mathrm{~cm},
$$

where $\alpha(r)$ becomes unity. For model $\mathrm{T}+\mathrm{C}$, we find $\alpha \approx$ $0.003, m=10$, and $\dot{m} \approx 5 \times 10^{-6}$, so $R_{0} \approx 144 r_{G}$ and $R_{1} \approx 3 r_{G}$. We therefore do not actually expect a full MDAF solution for this particular model. Instead we can make comparisons with the transition solution that applies between $R_{1}$ and $R_{0}$.

The analytic transitional flow model model predicts the following set of scaling relations for the disk properties (Meier 2005, 2008):

$$
\begin{aligned}
\rho_{c} & =2.1 \times 10^{-5} \alpha^{-1} \mathrm{~m}^{-1} \dot{m} x^{-3 / 2} \mathrm{~g} \mathrm{~cm}^{-3} \\
P_{c} & =7.4 \times 10^{12} \alpha^{-3 / 5} \mathrm{~m}^{-1} \dot{m}^{3 / 5} x^{-3 / 2} \mathrm{erg} \mathrm{cm}^{-3} \\
T_{c} & =2.65 \times 10^{9} \alpha^{2 / 5} \dot{m}^{-2 / 5} \mathrm{~K} \\
H & =4.4 \times 10^{4} \alpha^{1 / 5} \dot{m}^{-1 / 5} x^{3 / 2} \mathrm{~cm} \\
\alpha(x) & =5.5 \times 10^{3} \alpha^{2 / 5} \dot{m}^{3 / 5} x^{-3 / 2} \\
\beta(x) & =3.8 \times 10^{-9} \alpha^{2 / 5} \dot{m}^{-7 / 5} x^{7 / 2} \\
B^{r} & =2.21 \times 10^{11} \alpha^{-1 / 2} m^{-1 / 2} \dot{m} x^{-5 / 2} \mathrm{G} \\
r B^{\phi} & =2.3 \times 10^{6} \alpha^{3 / 10} \mathrm{~m}^{-1 / 2} \dot{m}^{1 / 5} x^{-1 / 2} \mathrm{G} \\
B^{Z} & =1.31 \times 10^{5} \alpha^{1 / 2} \mathrm{~m}^{-1 / 2} \mathrm{G} \\
V^{r} & =-1.62 \times 10^{11} \alpha^{4 / 5} \dot{m}^{1 / 5} x^{-1} \mathrm{~cm} \mathrm{~s}^{-1} \\
V_{A} & =1.36 \times 10^{13} \dot{m}^{1 / 2} x^{-7 / 4} \mathrm{~cm} \mathrm{~s}^{-1}
\end{aligned}
$$

where $x \equiv r / 6 r_{G}$ at the disk midplane.

Figures 3 and 4 above include the predictions for the MDAF transition region (equations 43) over the appropriate radial range, $R_{1}<r<R_{0}$. We find that the radiatively-cooled numerical simulation $\mathrm{T}+\mathrm{C}$ fits the MDAF transition solution remarkably well, except in certain circumstances.

First of all, Figure 3 shows that the density, pressure, and $\alpha(r)$ parameter are fit not only qualitatively but also quantitatively by the analytic transitional flow model, at least out to $R_{0} \approx 150 r_{G}$. The temperature structure, however, is a factor of 3 cooler than the analytic model and is not as constant with radius. This is due to the analytic model's assumption that generic Compton cooling dominates (i.e., $y \approx 1$ ) when, in fact, it is specifically Comptonized synchrotron cooling that is important in the simulations. The latter can have a slightly different value and depends additionally on the magnetic field strength, resulting in a slightly lower temperature that may not be constant with radius. This discrepancy also affects the disk scale height, which scales as $\sim \sqrt{T}$.

In Figure 4, again, most properties are fit well by the analytic model except for two: $B^{r}$ and $r B^{\phi}$. In the numerical simulation, while the general magnitude of the magnetic field (seen in $V_{A} / c$ ) fits fairly well (as does even the axial component $B^{Z}$ ), the distribution of the rest of the magnetic field into $r$ and $\phi$ components appears reversed from the analytic predictions. That is, the predicted flux conservation does not take place. There are different possible reasons for this:

- Any radial shear that could create $B^{r}$ from $r B^{\phi}$ is suppressed by the 2-D, axisymmetric nature of the simulations. 3-D simulations may show the expected distribution of $B^{r}$ and $B^{\phi}$ in the transition region.

- The natural state of magnetized accretion flow, even with cooling, may be like that of the RIAF models (e.g., $\mathrm{T}$ and I): always dominated by toroidal magnetic field. In this case, the predicted MDAF would not arise even with catastrophic cooling.

Therefore, it will be important to compare such 2-D simulations with similar 3-D ones to see how the inner transitional and predicted MDAF flows evolve when a third free dimension is added. Indeed, both toroidallydominated and radially-dominated flows may be possible in nature in this region, with a state transition between the two occurring from time-to-time.

\section{CONCLUSIONS}

Using the Cosmos++ code, we have performed twodimensional general relativistic MHD simulations of MRI-unstable accretion flows around black holes, with the potential of bremsstrahlung, synchrotron, and Compton cooling of the high-temperature inflow. In the process of implementing the radiative cooling processes in our code, we made the following observations:

- The cooling function in Esin et al. (1996), while valid in the range $10^{8} \mathrm{~K}<T<10^{11} \mathrm{~K}$, needs special attention and care in order to be valid outside that range and not lead to heating or freezing runaways in the simulations.

- If radiative cooling is to be added to MRI simulations, then the energy equation also must properly 
handle the "viscous" heating caused by reconnection and dissipation inherent in the MRI turbulence. In the present era of moderate-resolution MRI simulations (where dissipation is caused by numerical effects), this can be handled in one of two ways: perform total-energy-conserving simulations and compute the internal energy by subtracting the kinetic and magnetic energies from the total; or use an artificial resistivity term to resolve current sheets and allow energy lost through numerical reconnection to be recaptured as heat. Although the artificial resistivity technique has been used with good success in many Newtonian applications (e.g. Nitta et al. 2001, Stone \& Pringle 2001, Fragile et al. 2005), it has only recently been tested in a relativistic MHD code (Komissarov 2007).

- Indeed, evolving internal, rather than total, energy without an additional procedure for recapturing lost heat produces an unphysical numerical cooling which can rival, or exceed, true radiative cooling. Furthermore, even when the magnitude of cooling is comparable, the resulting disk structure is quite different for the internal-energy-only model.

If our assumption of $T_{e} \approx T_{i}$ is valid, then we obtain the following results pertaining to the astrophysics of radiatively cooled, magnetized accretion flows:

- Model $\mathrm{T}+\mathrm{C}$ confirms the "transitional flow" solution, which is proposed to connect an outer ADAFlike flow with an inner magnetically-dominated flow, as a viable MHD accretion inflow state.

- The accretion rate and magnetic viscosity parameter $\left(\dot{M} / \dot{M}_{E d d} \approx 5 \times 10^{-6}, \alpha \approx 0.003\right)$ that result from our choices of inputs are in a range that produces a large transitional flow region, without leading to a completely magnetically dominated state (i.e. $\alpha$ and $\beta^{-1}$ never exceed unity before the flow enters inside the last stable orbit).

- Comparison of the numerically-computed transitional flow and our prior analytic models of this region show remarkable qualitative and quantitative agreement. Exceptions are limited to the temperature structure of the analytic model (which used a cooling model much simpler than the numerical functions herein) and the radial vs. azimuthal magnetic structure (which likely was affected by the limitations of 2-dimensional axisymmetric MHD).

Further investigations into the development of a true MDAF solution will require additional 2-dimensional simulations to investigate inflows with smaller transitional and larger predicted true MDAF regions (i.e., with greater $\alpha$ and $\dot{M}$ ), and new 3-dimensional simulations to study the effects of cooling on the ratio of the radial to toroidal magnetic field components. The answers to these questions will determine whether or not radiative cooling ultimately can trigger the formation of large black hole coronae (MDAFs). These simulations may also help confirm that MDAFs can form moderate-speed jets as proposed in our introduction.

One final point should be noted. These are some of the first MRI simulations in which radiative cooling has an important dynamical effect on the accretion inflow. It was difficult, therefore, to predict what the resulting accretion rate and $\alpha$ parameter would be. The resulting values here $\left(\alpha=0.003, \dot{M}=5 \times 10^{-6}\right)$, did not turn out to be appropriate for a source in the upper righthand portion of the FBG diagram, as we had originally intended. In fact, they probably are more appropriate for a source in the lower right-hand portion with a rather low accretion rate. The applicability of these simulations to such a source, or really any source, will depend primarily on the validity of the assumption that $T_{e}=T_{i}$, as made in our cooling model.

We thank Sera Markoff and Masa Nakamura for their discussions and careful reading of this manuscript. We would like to recognize Joseph Niehaus for his contributions to testing the Cosmos++ code. PCF gratefully acknowledges the support of a Faculty R\&D grant from the College of Charleston and a REAP grant from the South Carolina Space Grant Consortium. Part of the research described in this paper was carried out at the Jet Propulsion Laboratory, California Institute of Technology, under contract to the National Aeronautics and Space Administration. Part of this research also was performed when the authors attended extended workshops at the UCLA Institute for Pure and Applied Mathematics and the UCSB Kavli Institute for Theoretical Physics. DLM is grateful to JPL/Caltech for financial support, and to UCLA for their hospitality during his sabbatical. This work also was supported by JPL subcontract 1304153 . Computing resources were provided by the JPL Supercomputing Facility and the Texas Advanced Computing Center (TACC). 


\section{REFERENCES}

Anninos, P., Fragile, P. C., \& Salmonson, J. D. 2005, ApJ, 635, 723

Beckwith, K., Hawley, J. F., \& Krolik, J. H. 2008, ApJ, 678, 1180

Begelman, M. C., \& Chiueh, T. 1988, ApJ, 332, 872

De Villiers, J., \& Hawley, J. F. 2003, ApJ, 589, 458

Esin, A. A., Narayan, R., Ostriker, E., \& Yi, I. 1996, ApJ, 465, 312

Fender, R. P., Belloni, T. M., \& Gallo, E. 2004, MNRAS, 355, 1105

Fragile, P. C., Anninos, P., Gustafson, K., \& Murray, S. D. 2005, ApJ, 619, 327

Hawley, J. F., Wilson, J. R., \& Smarr, L. L. 1984, ApJ, 277, 296

Hirose, S., Krolik, J. H., De Villiers, J.-P., \& Hawley, J. F. 2004, ApJ, 606, 1083

Hubeny, I. 1990, ApJ, 351, 632

Komissarov, S. S. 2007, MNRAS, 382, 995

Kozlowski, M., Jaroszynski, M., \& Abramowicz, M. A. 1978, A\&A, 63, 209

Mahadevan, R., Narayan, R., \& Yi, I. 1996, ApJ, 465, 327

McClintock, J. E., \& Remillard, R. A. 2006, Black hole binaries (Compact stellar X-ray sources), 157

McConnell, M. L., et al. 2002, ApJ, 572, 984

McKinney, J. C. 2006, MNRAS, 368, 1561

Meier, D. L. 2005, Ap\&SS, 300, 55
Meier, D. L. 2008, in preparation

Meier, D. L. 2009, Black Holes: The Ultimate Engines (in preparation)

Narayan, R., Yi, I., \& Mahadevan, R. 1995, Nature, 374, 623

Nitta, S., Tanuma, S., Shibata, K., \& Maezawa, K. 2001, ApJ, 550,1119

Noble, S. C., Leung, P. K., Gammie, C. F., \& Book, L. G. 2007, Classical and Quantum Gravity, 24, 259

Pacholczyk, A. G. 1970, Radio astrophysics. Nonthermal processes in galactic and extragalactic sources (Series of Books in Astronomy and Astrophysics, San Francisco: Freeman, 1970)

Shakura, N. I., \& Sunyaev, R. A. 1973, A\&A, 24, 337

Shapiro, S. L., Lightman, A. P., \& Eardley, D. M. 1976, ApJ, 204, 187

Sharma, P., Quataert, E., Hammett, G. W., \& Stone, J. M. 2007, ApJ, 667, 714

Stone, J. M., \& Pringle, J. E. 2001, MNRAS, 322, 461

Tomimatsu, A., \& Takahashi, M. 2001, ApJ, 552, 710

Uzdensky, D. A. 2004, ApJ, 603, 652

Uzdensky, D. A. 2005, ApJ, 620, 889

Wilson, J. R. 1972, ApJ, 173, 431

Yuan, F., Quataert, E., \& Narayan, R. 2003, ApJ, 598, 301 